\title{
The Self-Regulating Brain and Neurofeedback: Experimental Science and Clinical Promise
}

\author{
Robert T. Thibault ${ }^{\mathrm{a}}$, Michael Lifshitz ${ }^{\mathrm{a}}$, Amir Raz $^{\mathrm{a}, \mathrm{b}^{*}}$ \\ ${ }^{a}$ McGill University, 3775 University Street, Montreal, QC, H3A 2B4, Canada \\ ${ }^{\mathrm{b}}$ The Lady Davis Institute for Medical Research, 3755 Cote Ste. Catherine, Montreal, QC, \\ H3T 1E2, Canada
}

*Please address correspondence to:

Professor Amir Raz, 4333 Cote Ste. Catherine, Montreal, QC, H3T 1E4, Canada

amir.raz@mcgill.ca

Tel: 1-514-340-8210; Fax: 1-514-340-8124

(non-corresponding authors: robert.thibault@mail.mcgill.ca; michael.lifshitz2@mail.mcgill.ca) 


\begin{abstract}
Neurofeedback, one of the primary examples of self-regulation, designates a collection of techniques that train the brain and help to improve its function. Since coming on the scene in the 1960s, electroencephalography-neurofeedback has become a treatment vehicle for a host of mental disorders; however, its clinical effectiveness remains controversial. Modern imaging technologies of the living human brain (e.g., real-time functional magnetic resonance imaging) and increasingly rigorous research protocols that utilize such methodologies begin to shed light on the underlying mechanisms that may facilitate more effective clinical applications. In this paper we focus on recent technological advances in the field of human brain imaging and discuss how these modern methods may influence the field of neurofeedback. Toward this end, we outline the state of the evidence and sketch out future directions to further explore the potential merits of this contentious therapeutic prospect.
\end{abstract}

Keywords: self-regulation; neuroimaging; rtfMRI; psychiatry; neurofeedback. 


\section{INTRODUCTION}

Neurofeedback refers to a self-regulation technique that provides the individual with feedback about specific brain activity in connection with a related behavior. The underlying assumption at the core of this practice posits that through this type of feedback one can entrain, change, and regulate neural activity. This trend appeals to both researchers and practitioners, who wish to understand the neurobiological mechanisms as well as the therapeutic potential this approach may offer. Beyond electroencephalography (EEG), the advent of modern real-time brain imaging technology elucidates the time-course and location of brain activity and seems to open the road to new prospects, including the modulation of seemingly volitionless neural functions (Figure 1). And yet, imaging-based neurofeedback has hardly transitioned from the cognitive neuroscience lab into the clinical trenches. In this paper we highlight the relative merits and current shortcomings of neurofeedback in the context of contemporary imaging technologies. We discuss how new modalities of brain imaging may provide a future trajectory to consider meaningful research resulting in potential inclusion in the clinical armamentarium.

An evolutionary derivative of biofeedback, in the 1960s neurofeedback emerged to employ neural feedback via EEG (Kamiya, 2011). To this day, specialty clinics and private institutions continue to offer variations on EEG neurofeedback (EEG-nf) for an array of disorders and impairments, although this intervention has been largely dismissed as placebodriven (see next section). Beyond EEG-nf, the advent of new technologies for imaging the living human brain has vastly expanded the scope of neurofeedback, which today includes more novel methods such as functional magnetic resonance imaging (fMRI), functional near infrared spectroscopy (fNIRS), and magnetoencephalography (MEG) (TIMELINE and Table 1). Thus, current-day neurofeedback draws on diverse imaging methods to help drive volitional control 
over electromagnetic and hemodynamic alterations in brain activity (Cannon, 2015; Hammond, 2011; Thibault, Lifshitz, Birbaumer, \& Raz, 2015). Within each imaging modality, moreover, researchers have developed distinct neurofeedback protocols that target different brain signals and their concomitant physiological processes (Hammond, 2011; Sulzer, Haller, et al., 2013). Whereas proponents of neurofeedback sometimes lump together these diverse protocols, research findings support some imaging techniques more than others. In this paper we explore the potential merits and shortcomings of modern neurofeedback techniques and contextualize their place in the current technological landscape.

Figure 1. A conceptual diagram depicting rtfMRI-nf of the left primary motor cortex.

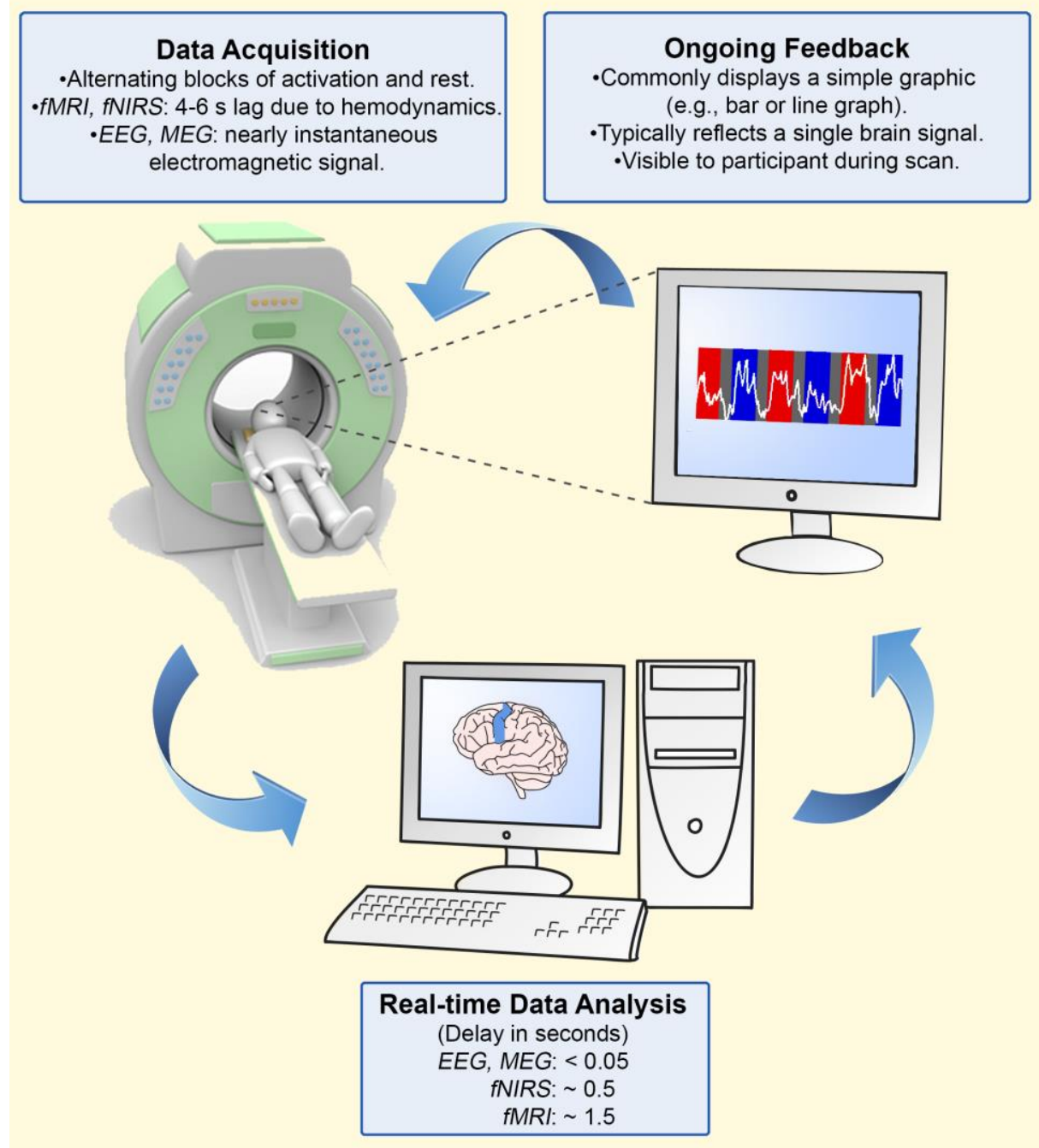


Timeline. A sketch of EEG-nf since its inception in the late 1950s. This visual representation captures the different organizations, journals, and events that shaped and fostered this technique. For over 40 years after the first neurofeedback experiment, EEG-nf dominated pertinent discussions. In 2003, however, real-time fMRI experiments sparked a new generation of research. References: 1. (Wyrwicka \& Sterman, 1968), 2. (Sterman \& Friar, 1972), 3. (Lubar \& Shouse, 1976), 4. (Weiskopf et al., 2003), 5. (Lal et al., 2005), 6. (deCharms et al., 2005) 7. (Sitaram, Zhang, et al., 2007), 8. (Buch et al., 2008), 9. (Mihara et al., 2012), 10. (Sulzer, Haller, et al., 2013), 11. (Zotev, Phillips, Yuan, Misaki, \& Bodurka, 2013), 12. (Arnold et al., 2013), 13. (Ogrim \& Hestad, 2013), 14. (Vollebregt, van Dongen-Boomsma, Buitelaar, \& Slaats-Willemse, 2014), 15. (Koush et al., 2013), 16. (Florin, Bock, \& Baillet, 2014), 17. (Okazaki et al., 2015), 18. (Marx et al., 2015), 19. (DeBettencourt, Cohen, Lee, Norman, \& Turk-Browne, 2015).

\section{Nearly six decades of neurofeedback $\square$ EEG-nf $\square$ fMRI-nf \\ $\square$ Emerging neurofeedback modalities}

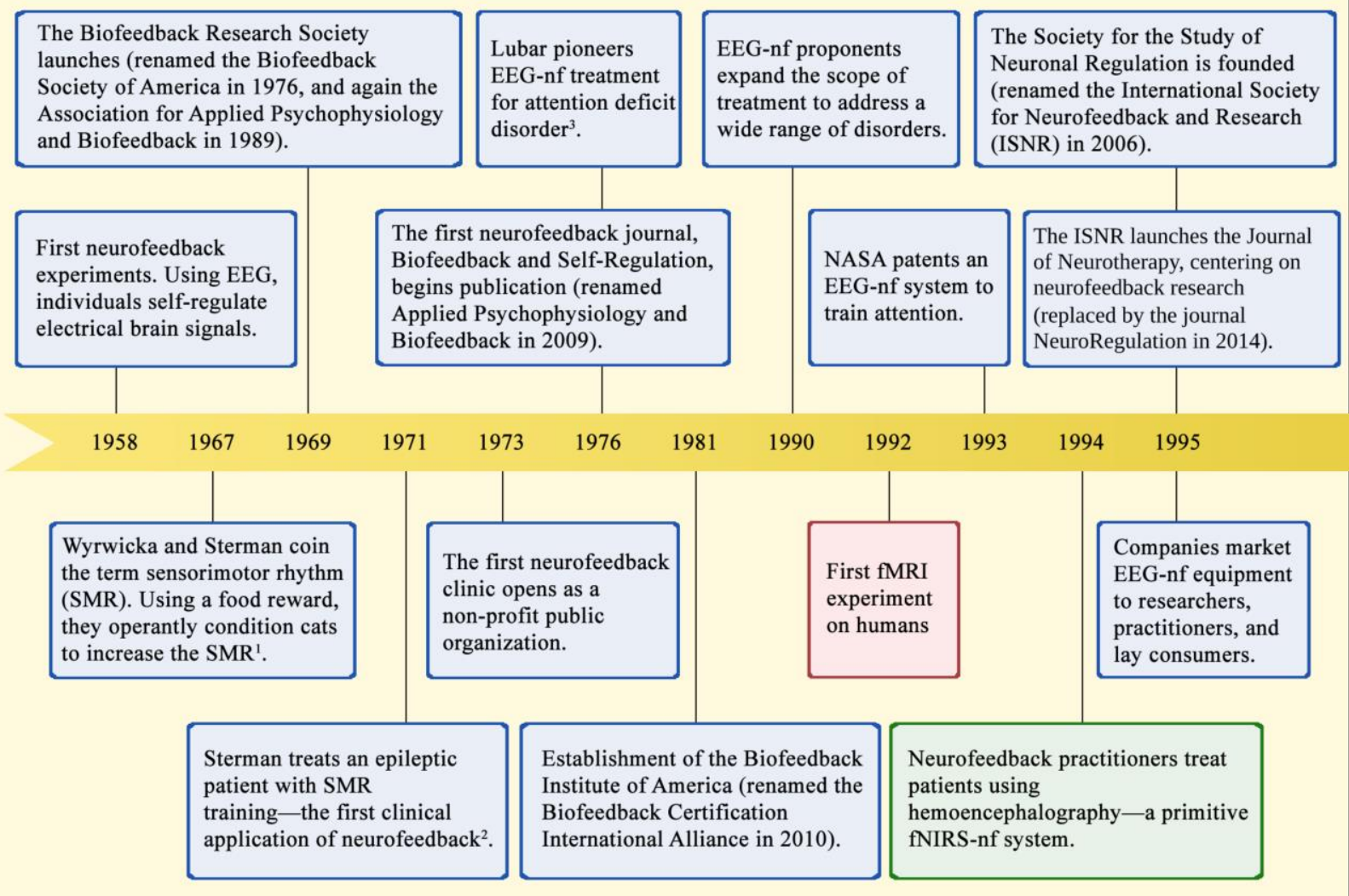




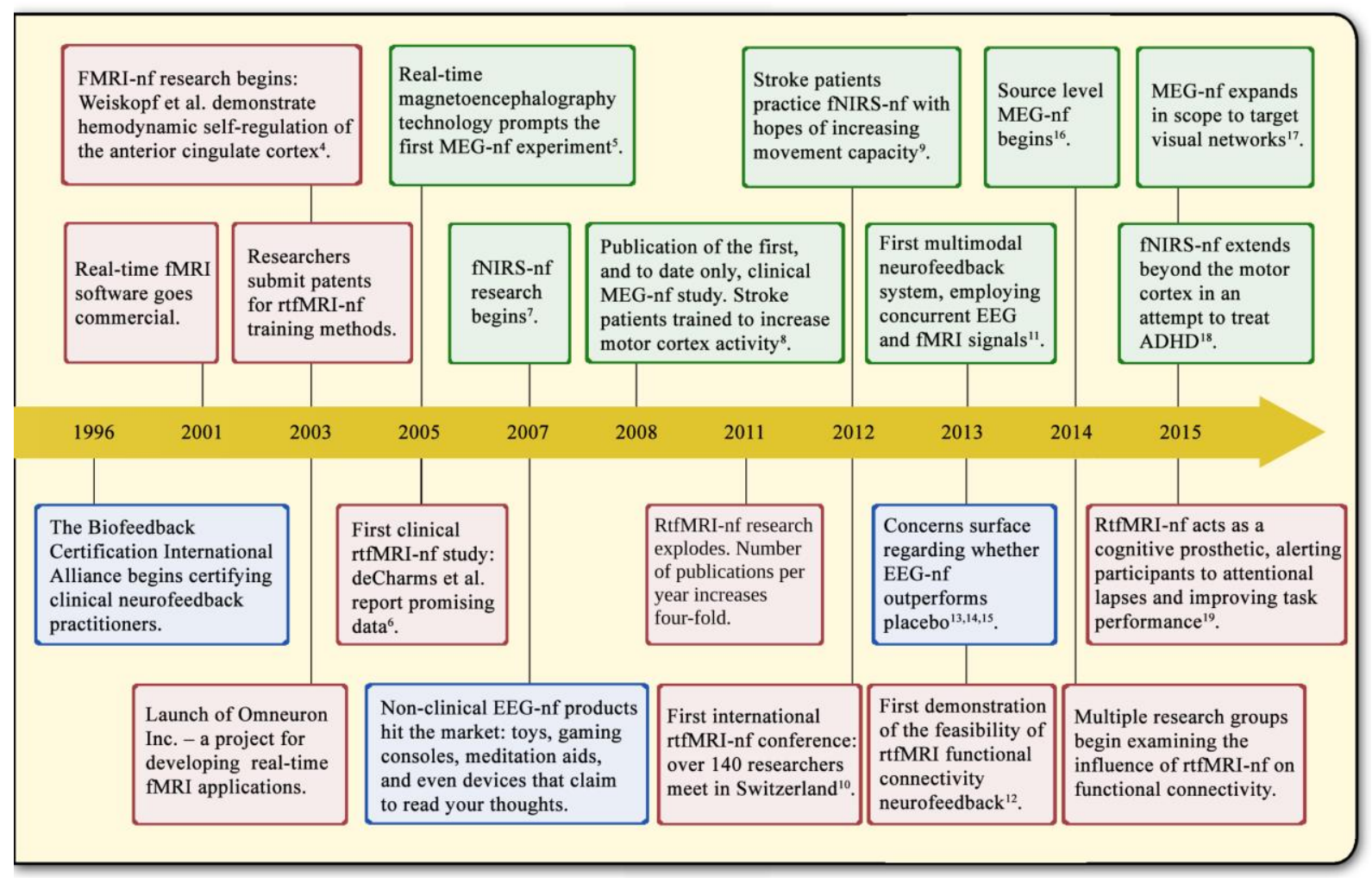

\section{EEG NEUROFEEDBACK}

More than half-a-century ago neurofeedback came on the scene promoting the main noninvasive technology of the day, EEG, to "image" the living human brain. Although EEG-nf may still hold some appeal as an alternative to conventional medical treatment, mounting evidence refutes the clinical superiority of feedback training over sham treatment (Arnold et al., 2013; Esmail \& Linden, 2014; Lansbergen et al., 2011; Lofthouse et al., 2012; Logeman et al., 2010; Perreau-Linck et al., 2010; Sonuga-Barke et al., 2013; Thibault, Lifshitz, Birbaumer, et al., 2015; Van Dongen-Boomsma et al., 2013; Vollebregt, van Dongen-Boomsma, Buitelaar, et al., 2014; Vollebregt, van Dongen-Boomsma, Slaats-Willemse, et al., 2014; Zuberer et al., 2015).

Nonetheless, EEG-nf remains a relatively active clinical field boasting international accreditation boards, specialized academic journals, and over a thousand practitioners (Thibault, Lifshitz, Birbaumer, et al., 2015). 
While EEG-nf proponents purport to effectively treat a range of psychological and neurological disorders (Arns, Heinrich, \& Strehl, 2014; Cannon, 2015; Hammond, 2011; Tan et al., 2009), it appears that influences other than the feedback itself bring about improvements in clinical endpoints across a range of disorders (Thibault, Lifshitz, Birbaumer, et al., 2015). For example, in the most researched application of EEG-nf - treatment of attention deficit hyperactivity disorder (ADHD) - all double-blind (Arnold et al., 2013; Lansbergen et al., 2011; Vollebregt, van Dongen-Boomsma, Buitelaar, et al., 2014), and multiple single-blind (PerreauLinck et al., 2010; Van Dongen-Boomsma et al., 2013), sham-controlled studies demonstrate comparable clinical outcomes in patients receiving veritable-feedback compared with shamfeedback. These studies often compare "relevant" information - i.e., genuine EEG-nf - with feedback from unrelated brain signals - e.g., neural activity from the brain of another participant, or a random signal (Box 1 depicts some of the pertinent ideas and terminology). Notably, double-blind sham-controlled studies, the gold-standard across most clinical research domains, are all but absent from clinical EEG-nf experiments beyond ADHD.

A number of reviews and meta-analyses have addressed the literature regarding EEG-nf treatment for ADHD (Arns et al., 2014; Arns, Ridder, \& Strehl, 2009; Lofthouse et al., 2012; Sonuga-Barke et al., 2013), epilepsy (Tan et al., 2009), depression and anxiety (Hammond, 2005), Parkinson's disease (Esmail \& Linden, 2014), and a slew of other disorders (Hammond, 2011). Some of these accounts highlight the dearth of convincing evidence (Esmail \& Linden, 2014; Lofthouse et al., 2012; Sonuga-Barke et al., 2013) while others rely on single-blinding, subpar control groups, non-peer-reviewed publications, and case studies to support EEG-nf (see Thibault et al., 2015 for a more in-depth exposition). The positive exception lies in one doubleblind sham-controlled study reporting greater clinical improvement in stroke patients receiving 
veritable rather than sham-feedback (Ramos-Murguialday et al., 2013). This state of affairs suggests that the benefits of EEG-nf for ADHD rely heavily on factors associated with demand characteristics and other contextual parameters rather than contingent feedback per se (Beyerstein, 1990; Thibault, Lifshitz, Birbaumer, et al., 2015). A scarcity of robust evidence precludes conclusions regarding the clinical efficacy of EEG-nf for epilepsy, depression, anxiety, addiction, Parkinson's disease, and stroke rehabilitation, among other disorders.

\section{Box 1: Important terminology}

Veritable feedback: Feedback from the region or frequency of interest (e.g., neural area or oscillation under study) derived directly from the participant's brain activity.

Sham feedback: Feedback not derived from the participant's brain activity. Or, feedback derived from the participant's brain, but not from the region or frequency of interest. Researchers occasionally give random feedback or feedback obtained from a previous trial with a different participant.

Specific treatment / Specificity: Treatments that work as a result of a precise, designated, mechanism of action.

Nonspecific factors: Factors that contribute to post-treatment changes but are not the result of the designated mechanism of action. These can include, but are not limited to, spontaneous remission and positive expectancy.

Reward contingency: When a particular behavior or outcome leads to a specific reward. Altering or reversing reward contingency, while maintaining all other elements of an experimental design, is an effective manner of testing treatment specificity.

Some proponents of EEG-nf argue that nonspecific factors alone cannot account for clinical improvement because changes are observable via objective measurements, including EEG (Leins et al., 2007; Strehl et al., 2006) and resting-state fMRI (Lévesque, Beauregard, \& Mensour, 2006; Ros et al., 2013). However, few, if any, studies rule out the influence of other parameters, which are intrinsic to feedback training (Box 2 provides a partial list). Moreover, nonspecific factors often propel highly specific changes in both behavior and neural activity (Raz 
\& Michels, 2007). In addition, many neurofeedback experiments employ either no control group or a control condition that substantively differs from the target intervention (e.g., in terms of length, intensity, and mode of training). And yet, EEG-nf may harbor a sizeable placebo component because it costs money, requires dozens of sessions, involves medical-like instrumentation, and carries the allure of brain science (Ali, Lifshitz, \& Raz, 2014). Add to that an emphasis on cognitive demands that often aims to improve psychological rather than physiological conditions (Benedetti et al., 2003; Moseley et al., 2002; Waber, Shiv, Carmon, \& Ariely, 2008) and the multi-faceted nature of neurofeedback becomes evident: whereas EEG-nf alters both brain patterns and behavioral measures, current findings hardly support a direct link between the specific feedback and these observed alterations.

\section{Box 2: Placebos}

Without a sham control group researchers cannot conclusively establish the degree to which the designated mechanism is responsible for patient improvement. Moreover, because neurofeedback involves many psychosocial elements, participants undergoing feedback training may be particularly susceptible to nonspecific factors. Over the past decade neurofeedback research has increasingly accounted for these ulterior parameters. Here is a sample:

Demand characteristics: In psychological experiments participants often show improvements which parallel the expectation of the experimenter, especially if they are explicitly aware of the experimental hypotheses (Nichols \& Maner, 2008).

Treated Condition: When administered a placebo, patients with disorders highly amenable to psychological factors show greater improvements than patients with disorders less amenable to psychological factors (Wampold, Minami, Tierney, Baskin, \& Bhati, 2005).

Interfacing with Technology: Individuals overestimate the capacity of seemingly cutting-edge technology (Ali et al., 2014), potentially increasing motivation and the expectation to improve.

Interacting with a practitioner: Simply meeting a clinician can instigate healing effects (Margo, 1999).

Price: Expensive treatments are more effective than less costly equivalents (Waber et al., 2008).

Intensity: More intensive medical procedures produce greater placebo effects (Kaptchuk et al., 2006).

Age: Children respond more to placebos than do adults (Rheims, Cucherat, Arzimanoglou, \& Ryvlin, 2008). 
Table 1: Advantages, shortcomings, and applications of neurofeedback imaging modalities

\begin{tabular}{|c|c|c|c|c|}
\hline & EEG & MEG & fMRI & fNIRS \\
\hline $\begin{array}{l}\text { Underlying } \\
\text { Signal }\end{array}$ & $\begin{array}{l}\text { Electrical activity } \\
\text { from pyramidal } \\
\text { cells perpendicular } \\
\text { to the scalp (mainly } \\
\text { gyri) }\end{array}$ & $\begin{array}{l}\text { Magnetic fields } \\
\text { produced by } \\
\text { pyramidal cells } \\
\text { perpendicular and } \\
\text { tangential to the } \\
\text { cortical surface }\end{array}$ & $\begin{array}{l}\text { Blood oxygenation } \\
\text { level dependent } \\
\text { contrast (which } \\
\text { indirectly relates } \\
\text { with neuronal } \\
\text { activity) }\end{array}$ & $\begin{array}{l}\text { Volume of } \\
\text { oxygenated and/or } \\
\text { deoxygenated blood } \\
\text { (which indirectly } \\
\text { relates with neuronal } \\
\text { activity) }\end{array}$ \\
\hline $\begin{array}{l}\text { Typical } \\
\text { Feedback } \\
\text { Signal Source }\end{array}$ & $\begin{array}{l}\text { One central } \\
\text { electrode or a } \\
\text { multi-electrode cap }\end{array}$ & $\begin{array}{l}\text { Sensors over } \\
\text { sensorimotor } \\
\text { cortex }\end{array}$ & $\begin{array}{l}\text { Single brain regions, } \\
3 \mathrm{~mm} \times 3 \mathrm{~mm} \text { voxels }\end{array}$ & $\begin{array}{l}\text { Several sensors over } \\
\text { sensorimotor cortex }\end{array}$ \\
\hline $\begin{array}{l}\text { Feedback } \\
\text { delay }\end{array}$ & $<50 \mathrm{~ms}$ & $<50 \mathrm{~ms}$ & $\begin{array}{l}\sim 1.5 \mathrm{~s} \text { (plus } 4-6 \mathrm{~s} \\
\text { hemodynamic delay) }\end{array}$ & $\begin{array}{l}\sim 0.5 \mathrm{~s} \text { (plus } 4-6 \mathrm{~s} \\
\text { hemodynamic delay) }\end{array}$ \\
\hline $\begin{array}{l}\text { Resolution } \\
\text { temporal } \\
\text { spatial } \\
\text { depth }\end{array}$ & $\begin{array}{l}\text { Milliseconds } \\
\text { Centimeters } \\
\text { Superficial }\end{array}$ & $\begin{array}{l}\text { Milliseconds } \\
\sim 10 \mathrm{~mm} \\
\text { Depth constrains } \\
\text { interpolation } \\
\text { accuracy }\end{array}$ & $\begin{array}{l}\text { Seconds } \\
\text { Millimeters } \\
\text { Deep (any region) }\end{array}$ & $\begin{array}{l}\text { Seconds } \\
\text { Centimeters } \\
\text { Superficial }(<4 \mathrm{~cm})\end{array}$ \\
\hline Portable & Yes & No & No & Yes \\
\hline $\begin{array}{l}\text { Cost (USD) } \\
\text { Initial set-up } \\
\text { Running costs }\end{array}$ & $\begin{array}{l}500-50,000 \\
\text { No extra fees }\end{array}$ & $\begin{array}{l}2,000,000 \\
\sim 500 / \text { hour }\end{array}$ & $\begin{array}{l}500,000-2,000,000 \\
\sim 500 / \text { hour }\end{array}$ & $\begin{array}{l}50,000-300,000 \\
\text { No extra fees }\end{array}$ \\
\hline $\begin{array}{l}\text { Relevant } \\
\text { Literature }\end{array}$ & Plentiful & Emerging & Moderate & Emerging \\
\hline $\begin{array}{l}\text { Main } \\
\text { Applications }\end{array}$ & $\begin{array}{l}\text { Pediatric ADHD, } \\
\text { epilepsy, various } \\
\text { psychological } \\
\text { disorders }\end{array}$ & $\begin{array}{l}\text { Brain computer } \\
\text { interfaces } \\
\text { (Experimental) }\end{array}$ & $\begin{array}{l}\text { Psychological } \\
\text { conditions (chronic } \\
\text { pain, depression, } \\
\text { schizophrenia, etc.) } \\
\text { (Experimental) }\end{array}$ & $\begin{array}{l}\text { Brain computer } \\
\text { interfaces, stroke } \\
\text { rehabilitation } \\
\text { (Experimental) }\end{array}$ \\
\hline
\end{tabular}




\section{3. fMRI}

Based on fMRI, real-time functional MRI neurofeedback (rtfMRI-nf) offers a noninvasive method to modulate regional neural activity. RtfMRI provides a way to collect, process, and display blood oxygen-level-dependent (BOLD) fMRI data within a short delay (e.g., 1-2 seconds; Weiskopf, 2012). Because of this nearly-concurrent acquisition-processing-display cycle, rtfMRI-nf training putatively permits individuals to see and thereby self-regulate fMRI signals from their own brains. Unlike other EEG-based neurofeedback, rtfMRI-nf training seems to yield precise localization and modulation of relevant brain structures (deCharms, 2008).

Whereas EEG-nf requires many sessions to alter electrical activity, with rtfMRI-nf individuals can selectively modify fMRI BOLD signal within 30 minutes of training and sometimes even sooner (Brühl et al., 2014; Canterberry et al., 2013; Greer, Trujillo, Glover, \& Knutson, 2014; Gröne et al., 2015; Hui, Zhang, Ge, Yao, \& Long, 2014; Lawrence et al., 2014; Paret et al., 2014; Sulzer, Sitaram, et al., 2013; Zhang, Zhang, et al., 2013). This striking difference between EEG-nf and rtfMRI-nf deserves further discussion. Learning based on neuro-hemodynamics may occur more quickly because unlike the absence of brain receptors to detect electrical changes, baroreceptors constantly inform the central nervous system about ongoing blood volume (Birbaumer, 2011). Some animal data (e.g., in rats) concerning baroreceptor activation support this speculative account (Dworkin, 1988). However, regardless of hypothetical insights (or lack thereof) regarding possible underlying mechanisms, humans seem to modulate neural hemodynamics faster than they do brain electrical fluctuations. This fact alone makes rtfMRI-nf an attractive vehicle to explore. 
The operationalization of rtfMRI-nf affords more focal investigations of higher (e.g., cognitive-behavioral) brain functions and neuroplasticity (deCharms et al., 2004; Weiskopf et al., 2007). For example, healthy individuals showed the ability to self-regulate brain activity in neuroanatomical structures often associated with affect (e.g., insula, amygdala, prefrontal cortex (PFC), and anterior cingulated cortex (ACC)) (Hamilton, Glover, Hsu, Johnson, \& Gotlib, 2011; Johnston et al., 2011; Posse et al., 2003; Sitaram, Caria, et al., 2007; Zotev et al., 2011). These findings poised rtfMRI-nf as a potentially effective intervention for several clinical domains, including pain regulation (e.g., deCharms et al., 2005), tinnitus (e.g., Haller, Birbaumer, \& Veit, 2010), Parkinson's disease (e.g., Subramanian et al., 2011) and depression (e.g., Linden et al., 2012; Young et al., 2014). Although these clinical studies represent mostly nascent efforts in line with pilot data and usually draw on small samples and largely unreplicated assays, the emerging tenor from these preliminary findings seems to speak favorably to the clinical potential of rtfMRI-nf. However, a careful evaluation of the literature reveals several caveats to quell this burgeoning assurance.

One of the early and most notable rtfMRI-nf studies implemented a careful design and reported robust findings that subsequently sparked considerable enthusiasm (deCharms et al., 2005). This study demonstrated that individuals who suffered from chronic pain and received veritable rtfMRI-nf learned to modulate neural activity in the rostral ACC. Moreover, these individuals were subsequently able to govern ACC activity even without feedback, resulting in the desired decreases in subjective pain. Because these effects were absent in control participants, this pivotal report presented important evidence in support of rtfMRI-nf. However, enthusiasm turned into skepticism after several independent replication efforts, including by the 
original authors, were unable to corroborate the reported findings (Birbaumer, Ruiz, \& Sitaram, 2013; Sulzer, Haller, et al., 2013).

One follow-up study included 59 chronic pain patients, rather than the 12 patients in the original study, and found that, while only those receiving veritable feedback gained control over neural activity, pain ratings decreased equally between experimental and sham participants (Sulzer, Haller, et al., 2013). The failure to replicate the original findings may relate to the inflated likelihood of false positives when employing small sample sizes - a general concern across the neurosciences (Button et al., 2013). Alternatively, some neurofeedback experts point out that in the replication effort, researchers provided participants in both the veritable feedback and control conditions with the same mental strategies — derived and optimized during previous neurofeedback experiments (Sulzer, Haller, et al., 2013). Thus, this attempt to replicate intimates that an effective mental strategy may show comparable effects regardless of whether one learns it through neurofeedback or through verbal instruction (Sulzer, Haller, et al., 2013). The impact of this key experiment, therefore, has been largely stripped of the promise it had originally heralded.

Most rtfMRI-nf accounts seldom probe behavioral change; instead, they report proof-ofconcept experiments demonstrating that participants can alter blood flow to select cortical regions. Out of over 70 published rtfMRI-nf studies, very few reported that participants were unable to modulate brain hemodynamics (Berman, Horovitz, Venkataraman, \& Hallett, 2011; Hampson et al., 2011). Other experiments using rtfMRI-nf, using sham-feedback as control, demonstrated that participants increased their ability to modulate a particular brain region throughout training (Caria et al., 2007; Caria, Sitaram, Veit, Begliomini, \& Birbaumer, 2010; Chiew, Laconte, \& Graham, 2012; Hui et al., 2014; Lawrence et al., 2014; McCaig, Dixon, 
Keramatian, Liu, \& Christoff, 2011; Rota et al., 2009; Rota, Handjaras, Sitaram, Birbaumer, \& Dogil, 2011; Yoo, Lee, O’Leary, Panych, \& Jolesz, 2008; Young et al., 2014; Zotev, Phillips, Young, Drevets, \& Bodurka, 2013; Zotev et al., 2011). Many other studies often lacked necessary controls or appropriate analyses to determine that veritable neurofeedback was the primary factor accounting for the observed brain alterations (Thibault et al., 2015); Figure 2 depicts some common control conditions. Thus, these collective findings raise some concerns about the specificity of neurofeedback, either sham or veritable. As a case in point, a recent study reported that participants receiving sham-neurofeedback expressed increased activation of bilateral insula, ACC, motor areas, and prefrontal areas (Ninaus et al., 2013) - arguably the four most common regions trained in rtfMRI-nf (Table 2). Experimental designs that lack a nofeedback or sham-feedback control cannot dissociate whether the change from baseline relies on feedback or on factors such as mental strategy and the attention and motivation that comes through participation in neurofeedback experiments. Thus, well-controlled designs are sorely missing, albeit desperately necessary, to tease apart the specific benefits of rtfMRI-nf.

The roles of "mental strategy" and "sustainability of effect" remain critical lacunae in the understanding of rtfMRI-nf. Mental strategy - the cognitive scheme one follows to achieve the desired goal - seems at least partly responsible for the rapid changes in fMRI BOLD signal. For example, a yet-to-be-published rtfMRI-nf experiment, discussed in an authoritative review (Sulzer, Haller, et al., 2013), showed that participants who received explicit instructions for mental strategies, compared to individuals left to explore and develop them implicitly on their own, were more successful at neural self-regulation. Moreover, comparing veritable feedback with no feedback (Zhang, Zhang, et al., 2013), inversely proportional feedback (Sulzer, Sitaram, et al., 2013), or feedback from a distinct brain region (Paret et al., 2014), revealed similar neural 
changes from baseline to the first trial in both experimental and control groups. On the other hand, findings from both human and animal studies suggest that explicit techniques are unnecessary and that contingent feedback alone is responsible for neural regulation (Caria et al., 2010; Koralek, Jin, Long, Costa, \& Carmena, 2012). Thus, the degree to which explicit mental strategies relate to learned brain modulation remains unclear.

\section{Neurofeedback Experimental Design $\square$ Proof-of-concept experiment $\square$ Application}

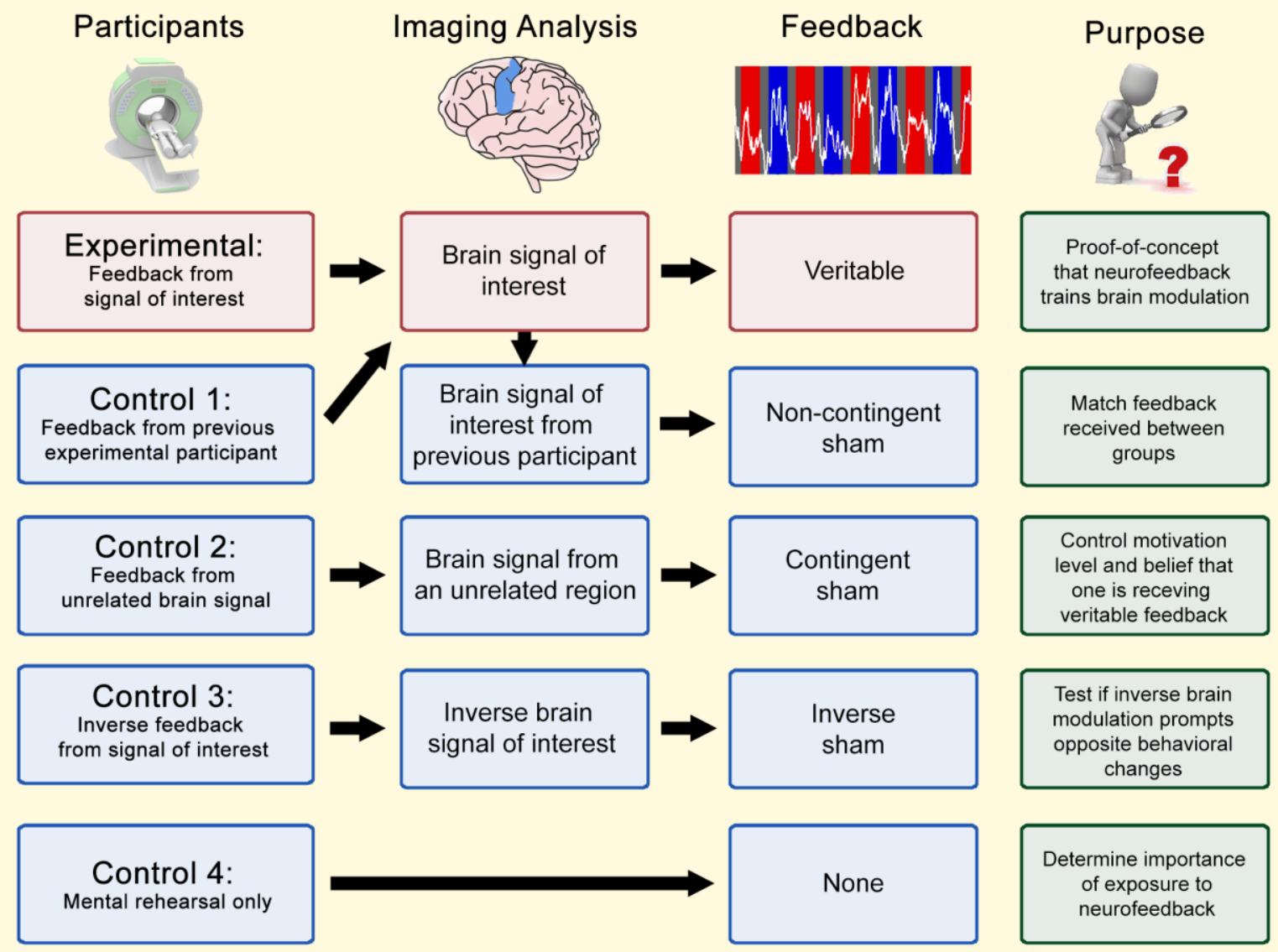

Figure 2. Robust experimental designs can better tease apart the effects of veritable neurofeedback and elucidate the underlying mechanisms driving neural regulation and altered behavior. This image depicts the format of many proof-of-concept neurofeedback studies (red) while outlining some of the most common control conditions (blue) and their relative merits (green). The most rigorous neurofeedback studies employ multiple control groups. 
In certain experiments, a minimum level of instruction may help participants avoid injurious strategies. For example, because both negative and positive emotions stimulate amygdala activation (e.g., Garavan, Pendergrass, Ross, Stein, \& Risinger, 2001; Hamann, Ely, Hoffman, \& Kilts, 2002; Yang et al., 2002), some experimenters take care to instruct participants to adopt positive mental strategies (Young et al., 2014; Zotev, Phillips, Young, Drevets, \& Bodurka, 2013). Similar considerations surface concerning up-regulation of the anterior insula, which responds strongly to disgust (e.g., Phillips et al., 1997; Wicker et al., 2003), and to modifying ACC activity, which relates to a wide spectrum of cognitive processes (e.g., Bush, Luu, \& Posner, 2000; Etkin, Egner, \& Kalisch, 2011). The potential for maladaptive mental strategies may present less of a concern for protocols aiming to modify sensorimotor areas through imagined movements.

Sustainability refers to the period of time, after feedback is over, during which participants can demonstrate the learned benefits of rtfMRI-nf. As it turns out, few studies examine sustainability in neurofeedback (Thibault et al., 2015). Whereas some experiments demonstrate that participants retain control over target brain regions (Caria et al., 2007; Mathiak et al., 2015; Robineau et al., 2014; Scharnowski et al., 2015; Zotev, Phillips, Young, et al., 2013; Zotev, Phillips, Yuan, et al., 2013; Zotev et al., 2011), other studies report no such retention (Berman, Horovitz, \& Hallett, 2013; Greer et al., 2014; Hamilton et al., 2011; Ruiz et al., 2013; Sulzer, Sitaram, et al., 2013). Thus, the conundrums of mental strategy and sustainability still beg resolution.

Relatively few accounts correlate rtfMRI-nf training with changes in either perception or behavior (Table 2). For example, two reports suggested that volitional dampening of ACC activity would decrease cigarette cravings (Canterberry et al., 2013; Li et al., 2012); however, 
both experiments feature deficient designs that make it difficult to determine what - e.g., feedback or mental strategy - actually accounted for the decreased desire to smoke (Thibault et al., 2015). Another example draws on analgesia: whereas one study demonstrated that modulation of ACC activity was independent of behavioral parameters such as perceived pain intensity (Rance, Ruttorf, Nees, Rudi Schad, \& Flor, 2014), another study found that control over activity in both the ACC and the anterior insula decreased pain perception (Emmert et al., 2014). According to some studies, modulating activity in the insula appears to influence the perceived valence of emotional stimuli (Caria et al., 2010; Ruiz et al., 2013); yet one of these studies had no control group (Ruiz et al., 2013) and another experiment suggested that control over activity in the insula area hardly related to valence ratings (Lawrence et al., 2014).

Beyond the ACC and insula, training motor areas can improve motor control in both healthy participants (Hui et al., 2014) and individuals diagnosed with Parkinson's disease (Subramanian et al., 2011); however, in the former study the control group also demonstrated improved finger-tapping speed while the latter experiment used no sham-feedback control condition. Altering prefrontal blood flow can improve detection of emotional prosodic intonations (Rota et al., 2009) and verbal working memory (Zhang, Yao, Zhang, Long, \& Zhao, 2013); however, again, the latter experiment demonstrated that sham-neurofeedback enhanced performance on four of the five working memory tasks, even while impairing the ability of participants to modulate target brain regions. On the other hand, control over brain activity in the amygdala appears to bear on emotional measures. Sham-controlled experiments demonstrated that successful amygdala modulation reduced ratings of anxiety and increased ratings of happiness in depressed patients (Young et al., 2014) and inversely correlated with difficulty identifying feelings and susceptibility to others' anger in healthy participants (Zotev et 
Table 2: Behavioral and clinical rtfMRI-nf experiments

\begin{tabular}{|c|c|c|}
\hline Brain Area & $\begin{array}{l}\text { Behavioral or } \\
\text { Clinical Target }\end{array}$ & Relevant rtfMRI-nf Experiments \\
\hline $\mathrm{ACC}$ & $\begin{array}{l}\text { Pain modulation } \\
\text { Smoking cessation } \\
\text { Emotional prosody } \\
\text { Cognitive interference }\end{array}$ & $\begin{array}{l}\text { deCharms et al. (2005), Emmert et al. (2014), } \\
\text { Rance et al. (2014), Guan et al. (2015) } \\
\text { Li et al. (2012), Canterberry et al. (2013) } \\
\text { Gröne et al. (2015) } \\
\text { Mathiak et al. (2015) }\end{array}$ \\
\hline Insula & $\begin{array}{l}\text { Emotional valence } \\
\text { Schizophrenia } \\
\text { Psychopathy } \\
\text { Pain modulation } \\
\text { Phobia }\end{array}$ & $\begin{array}{l}\text { Caria et al. (2010), Lawrence et al. (2014) } \\
\text { Ruiz et al. (2013), Cordes et al. (2015) } \\
\text { Sitaram et al. (2014) } \\
\text { Emmert et al. (2014), Rance et al. (2014) } \\
\text { Zilverstand et al. (2015) }\end{array}$ \\
\hline Motor Cortex & $\begin{array}{l}\text { Finger tapping speed } \\
\text { (Stroke rehabilitation) } \\
\text { Parkinson's disease } \\
\text { Reaction time } \\
\text { Precision grip }\end{array}$ & $\begin{array}{l}\text { Berman et al. (2011), Hui et al. (2014) } \\
\text { Subramanian et al. (2011) } \\
\text { Scharnowski et al. (2015) } \\
\text { Blefari et al. (2015) }\end{array}$ \\
\hline Amygdala & $\begin{array}{l}\text { Emotion identification } \\
\text { Depression }\end{array}$ & $\begin{array}{l}\text { Zotev et al. (2011) } \\
\text { Young et al. (2014) }\end{array}$ \\
\hline $\begin{array}{l}\text { Prefrontal } \\
\text { Cortex }\end{array}$ & $\begin{array}{l}\text { Emotional prosody } \\
\text { Depression } \\
\text { Working memory } \\
\text { Affect }\end{array}$ & $\begin{array}{l}\text { Rota et al. (2009) } \\
\text { Linden et al. (2012) } \\
\text { Zhang, Yao et al. (2013) } \\
\text { Sarkheil et al. (2015) }\end{array}$ \\
\hline Auditory cortex & Chronic tinnitus & Haller et al. (2010) \\
\hline Visual Cortex & Visual detection & Scharnowski et al. (2012) \\
\hline $\begin{array}{l}\text { Functional } \\
\text { Connectivity }\end{array}$ & Smoking cessation & Kim et al., (2015) \\
\hline
\end{tabular}

al., 2011). While neurofeedback may account for some of these outcomes, only experimental designs featuring more robust controls would permit ascertaining such specificity (Figure 2). Thus, at the end of the day, the literature provides a mixed bag with little by way of conclusive evidence. 
Recent studies have increasingly leveraged functional connectivity assays to index the effects of rtfMRI-nf (Emmert et al., 2014; Fukuda, Mitsuo, \& Hiroshi, 2015; Greer et al., 2014; Kim et al., 2015; Sarkheil et al., 2015; Shen, Zhang, Yao, \& Zhao, 2015; Sitaram et al., 2014; Zhang, Yao, Shen, Yang, \& Zhao, 2014). One study reported a link connecting functional connectivity, rtfMRI-nf, and behavior (Hui et al., 2014). Compared to sham-feedback controls, veritable neurofeedback altered brain connectivity in experimental participants: they improved their ability to modulate the premotor cortex and increased finger tapping speed (Hui et al., 2014). However, findings from other functional connectivity neurofeedback studies were less conclusive. Two analyses demonstrated post-training functional connectivity changes in participants receiving veritable feedback only, but experimental and control groups improved similarly on behavioral measures (Sarkheil et al., 2015; Shen et al., 2015). Thus, the overarching tenor emerging from these collective studies suggests that functional connectivity analyses scarcely bind neurofeedback with a change in behavior. If and when future experiments establish and cement this relationship, resorting to functional connectivity analysis may help unravel the neural mechanisms subserving self-regulation.

\section{4. fNIRS}

An emerging functional neuroimaging technology, fNIRS offers a relatively noninvasive, safe, and affordable way to monitor brain activity (Ferrari \& Quaresima, 2012). FNIRS technology typically uses a portable cap with approximately 50 channels to measure the concentration of deoxygenated blood in superficial brain areas. While fNIRS probes the same underlying hemodynamics as fMRI (Cui, Bray, Bryant, Glover, \& Reiss, 2011), each method 
possesses distinct strengths and weaknesses when applied to neurofeedback. On the one hand, fNIRS is relatively inexpensive, resistant to movement artifacts, and highly portable. On the other hand, whereas fNIRS offers spatial resolution on the order of centimeters at surface regions, fMRI provides millimetric precision throughout the brain (Table 1 provides a more detailed comparison).

Experiments with fNIRS-nf typically aim to show increased control over motor regions by having participants imagine tapping their fingers or clenching their hands. Four studies reported that fNIRS-nf increased activity in a variety of motor areas (Kanoh, Susila, \& Miyamoto, 2011; Kober et al., 2014; Mihara et al., 2012, 2013). However, simply moving a limb or covertly tensing a muscle can also modulate motor cortex activity and researchers rarely measure electromyography (EMG) to detect such covert muscle tensing. Thus, subtle, possibly subconscious, muscular activity may account for the observed increases in neural function. Proponents of fNIRS claim that this technique highlights how contingent feedback seems key: brain modules change activity as a function of genuine, but not sham, feedback (Kober et al., 2014; Mihara et al., 2013). However, veritable feedback may teach participants to, perhaps involuntarily and without awareness, increase muscle tension rather than develop new mental techniques. Thus, fNIRS-nf of the motor cortex may, in some ulterior fashion, operate as a form of EMG-biofeedback.

Beyond improvement over motor control, one fNIRS-nf experiment reported the successful regulation of prefrontal neural activity to treat ADHD (Marx et al., 2015). However, whereas participants who received fNIRS-nf improved on various measures of ADHD, comparable measures were apparent in controls participants, who received EEG-nf and EMGbiofeedback. Moreover, Marx et al. (2015) shy away from reporting whether participants 
learned to regulate the fNIRS signal. Thus, the link between improved symptomatology and fNIRS-nf remains tenuous.

\section{MEG}

MEG is an imaging modality that measures the amplitude of magnetic signals emitted from both superficial and deeper cortical areas. To record such activity, participants typically sit in a magnetically-shielded room while placing their head inside a helmet-shaped concave dewar, which features hundreds of magnetometers - sensors capable of detecting small changes in the magnetic field. Because such specialty apparatuses require an expensive set-up and substantial support facilities, MEG-nf largely remains the purview of brain researchers with access to advanced imaging centers. In line with EEG, MEG too provides a direct - rather than indirect (e.g., in the case of fMRI and fNIRS) - measure of neural activity. However, unlike with electrical signals, the skull and cephalic tissues hardly distort the magnetic field, thereby allowing magnetometers to accurately measure minute variations in neural activity. In addition, compared to other imaging modalities, MEG affords an impressive spatial resolution and signalto-noise ratio (Table 1 provides a detailed comparison).

Healthy participants using MEG-nf learned bidirectional control of neural activity, in a subset of the 9-15 $\mathrm{Hz}$ range over the motor cortex (often called the sensorimotor- or $\mu$-rhythm), within 64 minutes (Mellinger et al., 2007), and for neural sources involved in motor imagery in eight sessions of 12-18 minutes (Florin et al., 2014). However, as in the case for fNIRS-nf, here too participants may have unknowingly relied on limb muscles to increase cortical activation. This option receives further credence in findings coming from stroke patients, who manifest 
impaired muscular control and take much longer-13 to 22 sessions of one-to-two hours - to govern the MEG signal (Buch et al., 2008). Moreover, with one exception (Gallegos-Ayala et al., 2014), patients lacking control of all muscles (e.g., completely locked-in patients) have been entirely unsuccessful in maintaining control over neuroimaging signals (De Massari et al., 2013). Rather than neurofeedback, therefore, muscle tension - perhaps outside of common awareness may drive such improvements.

Two recent MEG-nf studies included behavioral measures and employed no-feedback (Boe et al., 2014) and sham-feedback (Okazaki et al., 2015) controls. One study demonstrated that only participants provided with feedback learned to increase source level activity at the primary sensorimotor cortex in three 40-minute sessions (Boe et al., 2014). This experiment included EMG sensors placed on the flexor and extensor muscles of the fingers-reducing, but not fully eliminating the potential of muscle contamination from bodily sources. Interestingly, both control and experimental groups improved finger dexterity equally on the only behavioral task included. The other experiment separated participants into two groups and provided half of each group with sham-feedback (Okazaki et al., 2015). Over a single 10-minute feedback session, one group attempted to increase posterior alpha lateralization to the left, while the other group aimed to lateralize the same activity to the right. One experimental group succeeded at increasing alpha lateralization and expressed an associated impairment in visual detection threshold. This account reported an absence of additional predicted changes in brain and behavior and scantily established a benefit to MEG-nf. Thus, whereas early MEG studies may have sparked excitement concerning new clinical prospects, more experiments would need to carefully control for the aforementioned caveats in order to verify the effectiveness of MEG-nf as a viable technique to modify brain and behavior. 


\section{FUTURE STEPS}

The breadth of neurofeedback techniques has greatly expanded with the advent of novel real-time imaging modalities. As we have discussed, however, the influence of neurofeedback on behavior remains largely indeterminate. Functional connectivity now pervades the neurofeedback literature as multi-modal imaging approaches the fore (Zotev, Phillips, Yuan, et al., 2013) and higher magnetic fields (e.g., MRI scanners operating at seven Tesla) rekindle enthusiasm amongst researchers (Gröne et al., 2015). Scientists are beginning to unravel how feedback training rearranges neural circuits; they may even begin to elucidate the feasibility of concurrent regulation over multiple distinct brain signals. And yet, for neurofeedback to earn the appellation of a solid intervention, we must establish the clinical significance of its purported therapeutic effects.

Whether feedback, rather than other ulterior factors, propels the neural changes requires further elucidation. Proof-of-concept neurofeedback experiments abound, yet few studies extend these efforts with more robust experimental paradigms. To establish whether a specific neurofeedback signal accounts for learned brain modulation, control conditions are imperative (Figure 2 depicts some key ideas). In addition to presenting control participants with shamfeedback, researchers should also aim to match less obvious factors between control and experimental groups. For example, some neurofeedback practitioners argue that the amount of positive feedback participants receive influences training outcome (Arns, Heinrich, \& Strehl, 2014).

Providing sham-feedback based on the brain activity from previously tested experimental participants would control for the level of positive feedback. Such control participants, however, 
may lose motivation if they experience an absence of control over the feedback signal and begin to believe they are receiving non-contingent feedback. Many clinical studies highlight that the majority of research participants can correctly guess their group assignments (i.e., experimental vs. placebo; Shapiro \& Shapiro, 1997). In some contexts, moreover, positive outcome relies more heavily on the belief of veritable treatment than on the actual treatment administered (Dar, Stronguin, \& Etter, 2005). Providing a second control group with feedback from a different area of their brain could help equalize the belief that they are receiving veritable feedback from the region of interest. To confirm the effectiveness of the control groups, researchers could collect phenomenological assays, including sense of control over the signal, motivation, and believed group assignments. Moreover, double-blind designs wherein both researchers and participants are unaware of group assignment would obviate any potential influence experimenters could unknowingly exert on participants (and vice versa). Such experimental designs hold the potential to thrust neurofeedback beyond the proof-of-principle stage while unraveling how ulterior factors, such as mental strategy and exposure to neurofeedback may contribute to neural self-regulation.

While more rigorous controls will likely help elucidate the putative relation between neurofeedback and brain modulation, additional conditions would further link feedback training to behavioral changes. For example, it would behoove neurofeedback proponents to study clinical populations and measure their behavior. In such experiments, researchers could provide some participants with inversely proportional feedback (i.e., present positive feedback to control participants for modulating the same signal of interest as the experimental group, yet in the opposing direction: thus, reversing the reward contingency (Box 1)). If inverse-controls and experimental participants learn to modulate brain signals in opposing directions while 
manifesting opposite behavioral changes, these findings would help cement the link between neurofeedback, brain self-regulation, and behavior.

Experimental designs leveraging sham control groups pose researchers with some ethical conundrums; from withholding effective treatment to engaging in potentially harmful procedures. Neurofeedback, however, has yet to amass the credence commensurate with the appellation of "evidence-based medicine" (Sulzer, Haller, et al., 2013; Thibault, Lifshitz, \& Raz, 2015). As such, employing sham controls falls short of qualifying as withholding effective treatment. Moreover, researchers employing sham controls could administer proper neurofeedback to all participants, after completion of the experiment, to ensure that all eventually receive veritable treatment - as is common in many clinical trials.

Implementing inverse feedback may appear unethical because it could theoretically worsen symptoms or impair behavior. And yet, the neurofeedback literature hardly supports these concerns. A few early EEG-nf experiments leveraged inverse controls for intractable epileptics (Lubar et al., 1981; Sterman \& MacDonald, 1978) and found comparable decreases in seizure frequency after veritable and inverse feedback sessions (Thibault, Lifshitz, Birbaumer, et al., 2015). As for rtfMRI-nf, only one study employs an inverse feedback control group and the authors do not report any adverse effects (Sulzer, Sitaram, et al., 2013). Interestingly, looking across experiments we find many cases of distinct protocols encouraging regulation in opposing directions. For example, many experiments train participants to up-regulate anterior insular activity to increase valence ratings (Caria, Sitaram, Veit, Begliomini, \& Birbaumer, 2010; Lawrence et al., 2014; Ruiz et al., 2013; Sitaram et al., 2014), while other studies encourage down-regulation to decrease pain perception (Emmert et al., 2014; Rance, Ruttorf, Nees, Rudi Schad, \& Flor, 2014) or subdue a phobia (Zilverstand, Sorger, Sarkheil, \& Goebel, 2015). 
Likewise, multiple experiments train down-regulation of the ACC to mitigate pain (deCharms et al., 2005; Guan et al., 2015) or inhibit cigarette cravings (Canterberry et al., 2013; Li et al., 2012), while others encourage ACC activity to heighten valence ratings (Gröne et al., 2015) or limit the effects of cognitive interference (Mathiak et al., 2010). Thus, researchers are already training regulation in opposite directions, but shy away from directly comparing the behavioral effects of up- versus down-regulation using a specific pattern of brain activity. To date, the reported negative effects of rtfMRI-nf plateau at mental fatigue and the kind of physical discomfort typical to brain imaging environments (Hawkinson et al., 2012; Sulzer, Haller, et al., 2013). In the absence of adverse reactions, therefore, ethical arguments against the use of inverse control conditions remain tenuous.

If well-controlled studies reinforce the connection between feedback, brain activity, and behavior, follow-up studies could then explore the feasibility of implementing such methods. The current literature concerning neurofeedback, however, largely evades the obligation of following circumspect experimental designs.

\section{CONCLUSION}

Neurofeedback is in vogue. Despite a vigorous revival over the past decade, recent accounts highlight a host of methodological and interpretational caveats that pervade the relevant scientific literature. Although neurofeedback draws on a diverse array of imaging methods, rudimentary questions regarding key concepts, such as sustainability and specificity, continue to linger largely unaddressed. While neurofeedback appears to help some participants gain the capacity for brain modulation, the relative contribution of specific feedback compared to ulterior 
factors remains unclear. At the moment, sparse behavioral measures, little follow-up sessions, and many methodological caveats preclude formal endorsement of neurofeedback as a clinical treatment vehicle. Although the jury is still out, additional judicious experiments and more compelling findings will have to further demonstrate the seductive, albeit yet unconfirmed, clinical promise of neurofeedback. 


\section{ACKNOWLEDGEMENTS}

Dr. Amir Raz acknowledges funding from the Canada Research Chair program (950-226108), Discovery (386156-2010) and Discovery Acceleration (396050-2010) Supplement grants from the Natural Sciences and Engineering Research Council of Canada (NSERC), Canadian Institutes of Health Research (MPE-117645), and the BIAL Foundation (118/14). Robert T. Thibault acknowledges a Fonds de recherche du Québec - Nature et technologies (FRQNT) graduate scholarship and an Alexander Graham Bell Canada Graduate Scholarship from NSERC. Michael Lifshitz acknowledges a Francisco J. Varela Research Award from the Mind and Life Institute and a Vanier Canada Graduate Scholarship from NSERC. We thank Drs. Xu Cui and Joseph Baker for providing the fNIRS photo. 


\section{REFERENCES}

Ali, S., Lifshitz, M., \& Raz, A. (2014). Empirical neuroenchantment: from reading minds to thinking critically. Frontiers in Human Neuroscience, 27(8), 357.

doi:http://dx.doi.org/10.3389/fnhum.2014.00357

Arnold, L. E., Lofthouse, N., Hersch, S., Pan, X., Hurt, E., Bates, B., ... Grantier, C. (2013). EEG neurofeedback for ADHD: double-blind sham-controlled randomized pilot feasibility trial. Journal of Attention Disorders, 17(5), 410-419. doi:10.1177/1087054712446173

Arns, M., Heinrich, H., \& Strehl, U. (2014). Evaluation of neurofeedback in ADHD: the long and winding road. Biological Psychology, 95, 108-15. doi:10.1016/j.biopsycho.2013.11.013

Arns, M., Ridder, S. De, \& Strehl, U. (2009). Efficacy of neurofeedback treatment in ADHD: the effects on inattention, impulsivity and hyperactivity: a meta-analysis. Clinical EEG and Neuroscience, 40(3), 180-189.

Benedetti, F., Pollo, A., Lopiano, L., Lanotte, M., Vighetti, S., \& Rainero, I. (2003). Conscious expectation and unconscious conditioning in analgesic, motor, and hormonal placebo/nocebo responses. The Journal of Neuroscience, 23(10), 4315-4323.

Berman, B. D., Horovitz, S. G., \& Hallett, M. (2013). Modulation of functionally localized right insular cortex activity using real-time fMRI-based neurofeedback. Frontiers in Human Neuroscience, 7, 1-11. doi:10.3389/fnhum.2013.00638

Berman, B. D., Horovitz, S. G., Venkataraman, G., \& Hallett, M. (2011). Self-modulation of primary motor cortex activity with motor and motor imagery tasks using real-time fMRIbased neurofeedback. NeuroImage, 59, 917-925. doi:10.1016/j.neuroimage.2011.07.035

Beyerstein, B. L. (1990). Brainscams: Neuromythologies of the New Age. International Journal of Mental Health Systems, 19(3), 27-36. doi:10.1186/1752-4458-3-7

Birbaumer, N. (2011). The Specificity of Feedback. Retrieved August 3, 2015, from http://www.gocognitive.net/interviews/specificity-feedback

Birbaumer, N., Ruiz, S., \& Sitaram, R. (2013). Learned regulation of brain metabolism. Trends in Cognitive Sciences, 17(6), 295-302. doi:10.1016/j.tics.2013.04.009

Blefari, M. L., Sulzer, J., Hepp-Reymond, M.-C., Kollias, S., \& Gassert, R. (2015). Improvement in precision grip force control with self-modulation of primary motor cortex during motor imagery. Frontiers in Behavioral Neuroscience, 9(February), 1-11. doi:10.3389/fnbeh.2015.00018 
Boe, S., Gionfriddo, A., Kraeutner, S., Tremblay, A., Little, G., \& Bardouille, T. (2014). Laterality of brain activity during motor imagery is modulated by the provision of source level neurofeedback. NeuroImage, 101, 159-167. doi:10.1016/j.neuroimage.2014.06.066

Brühl, A. B., Scherpiet, S., Sulzer, J., Stämpfli, P., Seifritz, E., \& Herwig, U. (2014). Real-time neurofeedback using functional MRI could improve down-regulation of amygdala activity during emotional stimulation: A proof-of-concept study. Brain Topography, 27, 138-148. doi:10.1007/s10548-013-0331-9

Buch, E., Weber, C., Cohen, L. G., Braun, C., Dimyan, M. a, Ard, T., ... Birbaumer, N. (2008). Think to move: a neuromagnetic brain-computer interface (BCI) system for chronic stroke. Stroke; a Journal of Cerebral Circulation, 39(3), 910-917. doi:10.1161/STROKEAHA.107.505313

Bush, G., Luu, P., \& Posner, M. I. (2000). Cognitive and emotional influences in anterior cingulate cortex. Trends in Cognitive Sciences, 4(6), 215-222. doi:10.1016/S13646613(00)01483-2

Button, K. S., Ioannidis, J. P. a, Mokrysz, C., Nosek, B. a, Flint, J., Robinson, E. S. J., \& Munafò, M. R. (2013). Power failure: why small sample size undermines the reliability of neuroscience. Nature Reviews. Neuroscience, 14(5), 365-76. doi:10.1038/nrn3475

Cannon, R. (2015). Editorial Perspective: Defining neurofeedback and its functional processes. NeuroRegulation, 2(2), 60-69. doi:10.15540/nr.2.2.60

Canterberry, M., Hanlon, C. A., Hartwell, K. J., Li, X., Owens, M., LeMatty, T., ... George, M. S. (2013). Sustained reduction of nicotine craving with real-time neurofeedback: exploring the role of severity of dependence. Nicotine \& Tobacco Research, 15(12), 2120-2124. doi:10.1093/ntr/ntt122

Caria, A., Sitaram, R., Veit, R., Begliomini, C., \& Birbaumer, N. (2010). Volitional control of anterior insula activity modulates the response to aversive stimuli. A real-time functional magnetic resonance imaging study. Biological Psychiatry, 68, 425-432. doi:10.1016/j.biopsych.2010.04.020

Caria, A., Veit, R., Sitaram, R., Lotze, M., Weiskopf, N., Grodd, W., \& Birbaumer, N. (2007). Regulation of anterior insular cortex activity using real-time fMRI. NeuroImage, 35, 12381246. doi:10.1016/j.neuroimage.2007.01.018

Chiew, M., Laconte, S. M., \& Graham, S. J. (2012). NeuroImage Investigation of fMRI neurofeedback of differential primary motor cortex activity using kinesthetic motor imagery. NeuroImage, 61(1), 21-31. doi:10.1016/j.neuroimage.2012.02.053

Cordes, J. S., Mathiak, K. A., Dyck, M., Alawi, E. M., Gaber, T. J., Zepf, F. D., ... Mathiak, K. (2015). Cognitive and neural strategies during control of the anterior cingulate cortex by 
fMRI neurofeedback in patients with schizophrenia. Frontiers in Behavioral Neuroscience, 9(June), 1-10. doi:10.3389/fnbeh.2015.00169

Cui, X., Bray, S., Bryant, D., Glover, G., \& Reiss, A. (2011). A quantitative comparison of NIRS and fMRI across multiple cognitive tasks. Neuroimage, 54(4), 2808-2821.

doi:10.1016/j.neuroimage.2010.10.069.A

Dar, R., Stronguin, F., \& Etter, J.-F. (2005). Assigned versus perceived placebo effects in nicotine replacement therapy for smoking reduction in Swiss smokers. Journal of Consulting and Clinical Psychology, 73(2), 350-353. doi:10.1037/0022-006X.73.2.350

De Massari, D., Ruf, C. A., Furdea, A., Matuz, T., van der Heiden, L., Halder, S., ... Birbaumer, N. (2013). Brain communication in the locked-in state. Brain : A Journal of Neurology, 136(Pt 6), 1989-2000. doi:10.1093/brain/awt102

DeBettencourt, M. T., Cohen, J. D., Lee, R. F., Norman, K. a, \& Turk-Browne, N. B. (2015). Closed-loop training of attention with real-time brain imaging. Nature Neuroscience, 18(3). doi:10.1038/nn.3940

deCharms, R. C. (2008). Applications of real-time fMRI. Nature Reviews. Neuroscience, 9(9), 720-729. doi:10.1038/nrn2414

deCharms, R. C., Christoff, K., Glover, G. H., Pauly, J. M., Whitfield, S., \& Gabrieli, J. D. . (2004). Learned regulation of spatially localized brain activation using real-time fMRI. NeuroImage, 21(1), 436-443. doi:10.1016/j.neuroimage.2003.08.041

deCharms, R. C., Maeda, F., Glover, G. H., Ludlow, D., Pauly, J. M., Soneji, D., .. Mackey, S. C. (2005). Control over brain activation and pain learned by using real-time functional MRI. Proceedings of the National Academy of Sciences of the United States of America, 102(51), 18626-31. doi:10.1073/pnas.0505210102

Dworkin, B. R. (1988). Hypertension as a learned response: The baroreceptor reinforcement hypothesis. In T. Elbert, W. Langosch, A. Steptoe, \& D. Vaitl (Eds.), Behavioral medicine in cardiovascular disorders (pp. 17-47). Chichester, UK: Wiley.

Emmert, K., Breimhorst, M., Bauermann, T., Birklein, F., Van De Ville, D., \& Haller, S. (2014). Comparison of anterior cingulate vs. insular cortex as targets for real-time fMRI regulation during pain stimulation. Frontiers in Behavioral Neuroscience, 8 (October), 350. doi:10.3389/fnbeh.2014.00350

Esmail, S., \& Linden, D. (2014). Neural Networks and Neurofeedback in Parkinson's Disease. NeuroRegulation, 1(3-4), 240-272. doi:10.15540/nr.1.3-4.240

Etkin, a, Egner, T., \& Kalisch, R. (2011). Emotional processing in anterior cingulate and medial prefrontal. Trends in Cognitive Sciences, 15(2), 85-93.

doi:10.1016/j.tics.2010.11.004.Emotional 
Ferrari, M., \& Quaresima, V. (2012). A brief review on the history of human functional nearinfrared spectroscopy (fNIRS) development and fields of application. NeuroImage, 63(2), 921-935. doi:10.1016/j.neuroimage.2012.03.049

Florin, E., Bock, E., \& Baillet, S. (2014). Targeted reinforcement of neural oscillatory activity with real-time neuroimaging feedback. NeuroImage, 88, 54-60.

doi:10.1016/j.neuroimage.2013.10.028

Fukuda, M., Mitsuo, K., \& Hiroshi, I. (2015). Functional MRI neurofeedback training on connectivity induces long-lasting changes in intrinsic functional network, 9(March). doi:10.3389/fnhum.2015.00160

Gallegos-Ayala, G., Furdea, A., Takano, K., Ruf, C. A., Flor, H., \& Birbaumer, N. (2014). Brain communication in a completeley locked-in patient using bedside near-infrared spectroscopy. Neurology, 82(21), 1930-32. doi:10.1111/j.1445-5994.2012.02896.x

Garavan, H., Pendergrass, J. C., Ross, T. J., Stein, E. a, \& Risinger, R. C. (2001). Amygdala response to both positively and negatively valenced stimuli. Neuroreport, 12(12), 27792783. doi:10.1097/00001756-200108280-00036

Greer, S. M., Trujillo, A. J., Glover, G. H., \& Knutson, B. (2014). Control of nucleus accumbens activity with neurofeedback. NeuroImage, 96, 237-244.

doi:10.1016/j.neuroimage.2014.03.073

Gröne, M., Dyck, M., Koush, Y., Bergert, S., Mathiak, K. A., Alawi, E. M., ... Mathiak, K. (2015). Upregulation of the Rostral Anterior Cingulate Cortex can Alter the Perception of Emotions: fMRI-Based Neurofeedback at 3 and 7 T. Brain Topography, 28, 197-207. doi:10.1007/s10548-014-0384-4

Guan, M., Li, L., Tong, L., Zhang, Y., Zheng, D., Yan, B., ... Shi, D. (2015). Self-regulation of rACC activation in patients with postherpetic neuralgia: A preliminary study using real-time fMRI neurofeedback. PloS One, 10(4), e0123675. doi:10.7910/DVN/27368

Haller, S., Birbaumer, N., \& Veit, R. (2010). Real-time fMRI feedback training may improve chronic tinnitus. European Radiology, 20(3), 696-703. doi:10.1007/s00330-009-1595-z

Hamann, S. B., Ely, T. D., Hoffman, J. M., \& Kilts, C. D. (2002). Ecstasy and Agony: Activation of the Human Amygdala in Positive and Negative Emotion. Psychological Science (WileyBlackwell), 13(2), 135.

Hamilton, J. P., Glover, G. H., Hsu, J.-J., Johnson, R. F., \& Gotlib, I. H. (2011). Modulation of subgenual anterior cingulate cortex activity with real-time neurofeedback. Human Brain Mapping, 32(1), 22-31. doi:10.1002/hbm.20997

Hammond, D. C. (2005). Neurofeedback Treatment of Depression and Anxiety. Journal of Adult Development, 12(2-3), 131-137. doi:10.1007/s 10804-005-7029-5 
Hammond, D. C. (2011). What is Neurofeedback: An Update. Journal of Neurotherapy, 15, 305-336. doi:10.1080/10874208.2011.623090

Hampson, M., Scheinost, D., Qiu, M., Bhawnani, J., Lacadie, C. M., Leckman, J. F., ...

Papademetris, X. (2011). Biofeedback of real-time functional magnetic resonance imaging data from the supplementary motor area reduces functional connectivity to subcortical regions. Brain Connectivity, 1(1), 91-8. doi:10.1089/brain.2011.0002

Hui, M., Zhang, H., Ge, R., Yao, L., \& Long, Z. (2014). Modulation of functional network with real-time fMRI feedback training of right premotor cortex activity. Neuropsychologia, 62, 111-123. doi:10.1016/j.neuropsychologia.2014.07.012

Johnston, S., Linden, D. E. J., Healy, D., Goebel, R., Habes, I., \& Boehm, S. G. (2011). Upregulation of emotion areas through neurofeedback with a focus on positive mood. Cognitive, Affective \& Behavioral Neuroscience, 11(1), 44-51. doi:10.3758/s13415-0100010-1

Kamiya, J. (2011). The First Communications About Operant Conditioning of the EEG. Journal of Neurotherapy, 15(1), 65-73. doi:10.1080/10874208.2011.545764

Kanoh, S., Susila, I., \& Miyamoto, K. (2011). The Effect of Neurofeedback Training on Cortical Activity during Motor Imagery Revealed by NIRS and fMRI. International Journal of Bioelectromagnetism, 13(2), 82-83.

Kaptchuk, T. J., Stason, W. B., Davis, R. B., Legedza, A. R. T., Schnyer, R. N., Kerr, C. E., ... Goldman, R. H. (2006). Sham device v inert pill: randomised controlled trial of two placebo treatments. $B M J, 1-7$.

Kim, D.-Y., Yoo, S.-S., Tegethoff, M., Meinlshmidt, G., \& Lee, J.-H. (2015). The Inclusion of Functional Connectivity Information into fMRI-based Neurofeedback Improves Its Efficacy in the Reduction of Cigarette Cravings. Journal of Cognitive Neuroscience, 27(8), 15521572. doi:10.1162/jocn

Kober, S. E., Wood, G., Kurzmann, J., Friedrich, E. V. C., Stangl, M., Wippel, T., ... Neuper, C. (2014). Near-infrared spectroscopy based neurofeedback training increases specific motor imagery related cortical activation compared to sham feedback. Biological Psychology, 95, 21-30. doi:10.1016/j.biopsycho.2013.05.005

Koralek, A. C., Jin, X., Long, J. D., Costa, R. M., \& Carmena, J. M. (2012). Corticostriatal plasticity is necessary for learning intentional neuroprosthetic skills. Nature, 483(7389), 331-5. doi:10.1038/nature10845

Koush, Y., Rosa, M. J., Robineau, F., Heinen, K., W Rieger, S., Weiskopf, N., ... Scharnowski, F. (2013). Connectivity-based neurofeedback: dynamic causal modeling for real-time fMRI. NeuroImage, 81(1), 422-30. doi:10.1016/j.neuroimage.2013.05.010 
Lal, T., Schröder, M., Hill, N., Preissl, H., Hinterberger, T., Mellinger, J., ... Schölkopf, B. (2005). A brain computer interface with online feedback based on magnetoencephalography. In Proceedings of the 22nd International Conference on Machine Learning. Bonn, Germany.

Lansbergen, M. M., van Dongen-Boomsma, M., Buitelaar, J. K., \& Slaats-Willemse, D. (2011). ADHD and EEG-neurofeedback: a double-blind randomized placebo-controlled feasibility study. Journal of Neural Transmission, 118(2), 275-84. doi:10.1007/s00702-010-0524-2

Lawrence, E. J., Su, L., Barker, G. J., Medford, N., Dalton, J., Williams, S. C. R., ... David, A. S. (2014). Self-regulation of the anterior insula: Reinforcement learning using real-time fMRI neurofeedback. NeuroImage, 88(1), 113-124. doi:10.1016/j.neuroimage.2013.10.069

Leins, U., Goth, G., Hinterberger, T., Klinger, C., Rumpf, N., \& Strehl, U. (2007). Neurofeedback for children with ADHD: a comparison of SCP and Theta/Beta protocols. Applied Psychophysiology and Biofeedback, 32, 73-88. doi:10.1007/s10484-007-9031-0

Lévesque, J., Beauregard, M., \& Mensour, B. (2006). neurofeedback training on the neural substrates of selective attention in children with attention-deficit/hyperactivity disorder: A functional magnetic resonance imaging. Neuroscience Letters, 301(3), 45-48. doi:10.1016/j.neulet.2005.10.100

Li, X., Hartwell, K. J., Borckardt, J., Prisciandaro, J. J., Saladin, M. E., Morgan, P. S., ... George, M. S. (2012). Volitional reduction of anterior cingulate cortex activity produces decreased cue craving in smoking cessation: a preliminary real-time fMRI study. Addiction Biology, 1-10.

Linden, D. E. J., Habes, I., Johnston, S. J., Linden, S., Tatineni, R., Subramanian, L., ... Goebel, R. (2012). Real-time self-regulation of emotion networks in patients with depression. PloS One, 7(6), e38115. doi:10.1371/journal.pone.0038115

Lofthouse, N., Arnold, L. E., Hersch, S., Hurt, E., \& DeBeus, R. (2012). A review of neurofeedback treatment for pediatric ADHD. Journal of Attention Disorders, 16(5), 35172. doi:10.1177/1087054711427530

Logeman, H. N. A., Lansbergen, M. M., van Os, T. W. D. P., Bocker, K. B. E., \& Kenemans, J. L. (2010). The effectiveness of EEG-feedback on attention, impulsivity and EEG: a sham feedback controlled study. Neuroscience Letters, 479, 49-53.

Lubar, J. F., Shabsin, H. S., Natelson, S. E., Holder, G. S., Whitsett, S. F., Pamplin, W. E., \& Krulikowski, D. I. (1981). EEG operant conditioning in intractable epileptics. Archives of Neurology, 38(11), 700-4.

Lubar, J. F., \& Shouse, M. N. (1976). EEG and behavioral changes in a hyperkinetic child concurrent with training of the sensorimotor rhythm (SMR): a preliminary report. Biofeedback and Self-Regulation, 1(3), 293-306. 
Margo, C. E. (1999). The Placebo Effect. Survey of Ophthamology, 44(1), 31-44.

Marx, A., Ehlis, A., Furdea, A., Holtmann, M., Banaschewski, T., Brandeis, D., ... Strehl, U. (2015). Near-infrared spectroscopy ( NIRS ) neurofeedback as a treatment for children with attention deficit hyperactivity disorder ( ADHD ) - a pilot study. Frontiers in Behavioral Neuroscience, 8(January), 1-13. doi:10.3389/fnhum.2014.01038

Mathiak, K. A., Alawi, E. M., Koush, Y., Dyck, M., Cordes, J. S., Gaber, T. J., ... Mathiak, K. (2015). Social reward improves the voluntary control over localized brain activity in fMRIbased neurofeedback training. Frontiers in Behavioral Neuroscience, 9(June), 136. doi:10.3389/fnbeh.2015.00136

Mathiak, K. A., Koush, Y., Dyck, M., Gaber, T. J., Alawi, E., Zepf, F. D., ... Mathiak, K. (2010). Social reinforcement can regulate localized brain activity. European Archives of Psychiatry and Clinical Neuroscience, 260, 132-136. doi:10.1007/s00406-010-0135-9

McCaig, R. G., Dixon, M., Keramatian, K., Liu, I., \& Christoff, K. (2011). Improved modulation of rostrolateral prefrontal cortex using real-time fMRI training and meta-cognitive awareness. NeuroImage, 55(3), 1298-305. doi:10.1016/j.neuroimage.2010.12.016

Mellinger, J., Schalk, G., Braun, C., Preissl, H., Rosenstiel, W., Birbaumer, N., \& Kübler, A. (2007). An MEG-based brain-computer interface (BCI). NeuroImage, 36(3), 581-593. doi:10.1016/j.neuroimage.2007.03.019

Mihara, M., Hattori, N., Hatakenaka, M., Yagura, H., Kawano, T., Hino, T., \& Miyai, I. (2013). Near-infrared Spectroscopy-mediated Neurofeedback Enhances Efficacy of Motor Imagerybased Training in Poststroke Victims: A Pilot Study. Stroke, 1-8.

Mihara, M., Miyai, I., Hattori, N., Hatakenaka, M., Yagura, H., Kawano, T., ... Kubota, K. (2012). Neurofeedback using real-time near-infrared spectroscopy enhances motor imagery related cortical activation. PloS One, 7(3), e32234. doi:10.1371/journal.pone.0032234

Moseley, J. B., O’Malley, K., Petersen, N. J., Menke, T. J., Brody, B. A., Kuykendall, D. H., ... Wray, N. P. (2002). A controlled trial of arthroscopic surgery for osteoarthritis of the knee. The New England Journal of Medicine, 347(2), 81-88.

Nichols, A. L., \& Maner, J. K. (2008). The Good-Subject Effect : Investigating Participant Demand Characteristics The Good-Subject Effect : Investigating Participant Demand Characteristics. The Journal of General Psychology, 135(2), 151-166.

Ninaus, M., Kober, S. E., Witte, M., Koschutnig, K., Stangl, M., Neuper, C., \& Wood, G. (2013). Neural substrates of cognitive control under the belief of getting neurofeedback training. Frontiers in Human Neuroscience, 7(December), 1-10. doi:10.3389/fnhum.2013.00914 
Ogrim, G., \& Hestad, K. A. (2013). Effects of neurofeedback versus stimulant medication in attention-deficit/hyperactivity disorder: a randomized pilot study. Journal of Child and Adolescent Psychopharmacology, 23(7), 448-57. doi:10.1089/cap.2012.0090

Okazaki, Y. O., Horschig, J. M., Luther, L., Oostenveld, R., Murakami, I., \& Jensen, O. (2015). NeuroImage Real-time MEG neurofeedback training of posterior alpha activity modulates subsequent visual detection performance. NeuroImage, 107, 323-332. doi:10.1016/j.neuroimage.2014.12.014

Paret, C., Klütsch, R., Ruf, M., Demirakca, T., Hösterey, S., Ende, G., \& Schmahl, C. (2014). Down-regulation of amygdala activation with real-time fMRI neurofeedback in a healthy female sample. Frontiers in Behavioral Neuroscience, 8(September), 1-15. doi:10.3389/fnbeh.2014.00299

Perreau-Linck, E., Lessard, N., Lévesque, J., \& Beauregard, M. (2010). Effects of Neurofeedback Training on Inhibitory Capacities in ADHD Children: A Single-Blind, Randomized, Placebo-Controlled Study. Journal of Neurotherapy, 14(3), 229-242. doi:10.1080/10874208.2010.501514

Phillips, M. L., Young, a W., Senior, C., Brammer, M., Andrew, C., Calder, a J., ... David, a S. (1997). A specific neural substrate for perceiving facial expressions of disgust. Nature, 389(October), 495-498. doi:10.1038/39051

Posse, S., Fitzgerald, D., Gao, K., Habel, U., Rosenberg, D., Moore, G. J., \& Schneider, F. (2003). Real-time fMRI of temporolimbic regions detects amygdala activation during single-trial self-induced sadness. NeuroImage, 18(3), 760-768. doi:10.1016/S10538119(03)00004-1

Ramos-Murguialday, A., Broetz, D., Rea, M., Läer, L., Yilmaz, O., Brasil, F. L., ... Birbaumer, N. (2013). Brain-machine interface in chronic stroke rehabilitation: a controlled study. Annals of Neurology, 74(1), 100-8. doi:10.1002/ana.23879

Rance, M., Ruttorf, M., Nees, F., Rudi Schad, L., \& Flor, H. (2014). Real time fMRI feedback of the anterior cingulate and posterior insular cortex in the processing of pain. Human Brain Mapping, 5798(July), 5784-5798. doi:10.1002/hbm.22585

Raz, A., \& Michels, R. (2007). Contextualizing specificity: Specific and non-specific effects of treatment. American Journal of Clinical Hypnosis, (October), 177-182. doi:http://dx.doi.org/10.1080/00029157.2007.10401614

Rheims, S., Cucherat, M., Arzimanoglou, A., \& Ryvlin, P. (2008). Greater response to placebo in children than in adults: a systematic review and meta-analysis in drug-resistant partial epilepsy. PLoS Medicine, 5(8), e166. doi:10.1371/journal.pmed.0050166

Robineau, F., Rieger, S. W., Mermoud, C., Pichon, S., Koush, Y., Van De Ville, D., ... Scharnowski, F. (2014). Self-regulation of inter-hemispheric visual cortex balance through 
real-time fMRI neurofeedback training. NeuroImage, 100, 1-14.

doi:10.1016/j.neuroimage.2014.05.072

Ros, T., Théberge, J., Frewen, P. A., Kluetsch, R., Densmore, M., Calhoun, V. D., \& Lanius, R. A. (2013). Mind over chatter: plastic up-regulation of the fMRI salience network directly after EEG neurofeedback. NeuroImage, 65, 324-35. doi:10.1016/j.neuroimage.2012.09.046

Rota, G., Handjaras, G., Sitaram, R., Birbaumer, N., \& Dogil, G. (2011). Reorganization of functional and effective connectivity during real-time fMRI-BCI modulation of prosody processing. Brain and Language, 117(3), 123-32. doi:10.1016/j.bandl.2010.07.008

Rota, G., Sitaram, R., Veit, R., Erb, M., Weiskopf, N., Dogil, G., \& Birbaumer, N. (2009). Selfregulation of regional cortical activity using real-time fMRI: the right inferior frontal gyrus and linguistic processing. Human Brain Mapping, 30(5), 1605-14. doi:10.1002/hbm.20621

Ruiz, S., Lee, S., Soekadar, S. R., Caria, A., Veit, R., Kircher, T., ... Sitaram, R. (2013). Acquired self-control of insula cortex modulates emotion recognition and brain network connectivity in schizophrenia. Human Brain Mapping, 34(1), 200-12. doi:10.1002/hbm.21427

Sarkheil, P., Zilverstand, A., Kilian-Hütten, N., Schneider, F., Goebel, R., \& Mathiak, K. (2015). fMRI feedback enhances emotion regulation as evidenced by a reduced amygdala response. Behavioural Brain Research, 281(0), 326-332. doi:http://dx.doi.org/10.1016/j.bbr.2014.11.027

Scharnowski, F., Hutton, C., Josephs, O., Weiskopf, N., \& Rees, G. (2012). Improving visual perception through neurofeedback. The Journal of Neuroscience, 32(49), 17830-41. doi:10.1523/JNEUROSCI.6334-11.2012

Scharnowski, F., Veit, R., Zopf, R., Studer, P., Bock, S., Göbel, R., ... Weiskopf, N. (2015). Manipulating motor performance and memory through neurofeedback. Biological Psychology, 108, 1-34. doi:http://dx.doi.org/10.1016/j.biopsycho.2015.03.009

Shapiro, A. K., \& Shapiro, E. (1997). How Blind is Blind. In The Powerful Placebo: From Ancient Priest to Modern Physician (pp. 190-216). John Hopkins University Press.

Shen, J., Zhang, G., Yao, L., \& Zhao, X. (2015). Real-time fMRI training-induced changes in regional connectivity mediating verbal working memory behavioral performance. Neuroscience, 289, 144-152. doi:10.1016/j.neuroscience.2014.12.071

Sitaram, R., Caria, A., Veit, R., Gaber, T., Rota, G., Kuebler, A., \& Birbaumer, N. (2007). FMRI brain-computer interface: a tool for neuroscientific research and treatment. Computational Intelligence and Neuroscience, 2007, 25487. doi:10.1155/2007/25487 
Sitaram, R., Caria, A., Veit, R., Gaber, T., Ruiz, S., \& Birbaumer, N. (2014). Volitional control of the anterior insula in criminal psychopaths using real-time fMRI neurofeedback : A pilot study, 8(October), 1-13. doi:10.3389/fnbeh.2014.00344

Sitaram, R., Zhang, H., Guan, C., Thulasidas, M., Hoshi, Y., Ishikawa, A., ... Birbaumer, N. (2007). Temporal classification of multichannel near-infrared spectroscopy signals of motor imagery for developing a brain-computer interface. NeuroImage, 34(4), 1416-27. doi:10.1016/j.neuroimage.2006.11.005

Sonuga-Barke, E. J. S., Brandeis, D., Cortese, S., Daley, D., Ferrin, M., Holtmann, M., ... Sergeant, J. (2013). Nonpharmacological interventions for ADHD: systematic review and meta-analyses of randomized controlled trials of dietary and psychological treatments. The American Journal of Psychiatry, 170(3), 275-89. doi:10.1176/appi.ajp.2012.12070991

Sterman, M., \& Friar, L. (1972). Suppression of seizures in an epileptic following sensorimotor EEG feedback training. Electroencephalography and Clinical Neurophysiology, 33, 89-95.

Sterman, M., \& MacDonald, L. (1978). Effects of central cortical EEG feedback training on incidence of poorly controlled seizures. Epilepsia, 19, 207-222.

Strehl, U., Leins, U., Goth, G., Klinger, C., Hinterberger, T., \& Birbaumer, N. (2006). Selfregulation of slow cortical potentials: a new treatment for children with attentiondeficit/hyperactivity disorder. Pediatrics, 118(5), e1530-40. doi:10.1542/peds.2005-2478

Subramanian, L., Hindle, J. V, Johnston, S., Roberts, M. V, Husain, M., Goebel, R., \& Linden, D. (2011). Real-time functional magnetic resonance imaging neurofeedback for treatment of Parkinson's disease. The Journal of Neuroscience, 31(45), 16309-17. doi:10.1523/JNEUROSCI.3498-11.2011

Sulzer, J., Haller, S., Scharnowski, F., Weiskopf, N., Birbaumer, N., Blefari, M. L., ... Sitaram, R. (2013). Real-time fMRI neurofeedback: progress and challenges. NeuroImage, 76(1), 386-99. doi:10.1016/j.neuroimage.2013.03.033

Sulzer, J., Sitaram, R., Blefari, M. L., Kollias, S., Birbaumer, N., Stephan, K. E., ... Gassert, R. (2013). Neurofeedback-mediated self-regulation of the dopaminergic midbrain. NeuroImage, 83(1), 817-25. doi:10.1016/j.neuroimage.2013.05.115

Tan, G., Thornby, J., Hammond, D. C., Strehl, U., Canady, B., Arnemann, K., \& Kaiser, D. A. (2009). Meta-Analysis of EEG Biofeedback in Treating Epilepsy. Clinical EEG and Neuroscience, 40(3), 173-179. doi:10.1177/155005940904000310

Thibault, R. T., Lifshitz, M., Birbaumer, N., \& Raz, A. (2015). Neurofeedback, Self-Regulation, and Brain Imaging : Clinical Science and Fad in the Service of Mental Disorders. Psychotherapy and Psychosomatics, 84(4), 193-207. doi:10.1159/000371714 
Van Dongen-Boomsma, M., Vollebregt, M. A., Slaats-Willemse, D., \& Buitelaar, J. K. (2013). A randomized placebo-controlled trial of electroencephalographic (EEG) neurofeedback in children with attention-deficit/hyperactivity disorder. Journal of Clinical Psychiatry, 74(August), 821-827. doi:10.4088/JCP.12m08321

Vollebregt, M. A., van Dongen-Boomsma, M., Buitelaar, J. K., \& Slaats-Willemse, D. (2014). Does EEG-neurofeedback improve neurocognitive functioning in children with attentiondeficit/hyperactivity disorder? A systematic review and a double-blind placebo-controlled study. Journal of Child Psychology and Psychiatry, and Allied Disciplines, 55(5), 460-72. doi:10.1111/jcpp.12143

Waber, R., Shiv, B., Carmon, Z., \& Ariely, D. (2008). Commercial features of placebo and therapeutic efficacy. Journal of the American Medical Association, 299(9), 1016-17.

Wampold, B. E., Minami, T., Tierney, S. C., Baskin, T. W., \& Bhati, K. S. (2005). The placebo is powerful: estimating placebo effects in medicine and psychotherapy from randomized clinical trials. Journal of Clinical Psychology, 61(7), 835-54. doi:10.1002/jclp.20129

Weiskopf, N. (2012). Real-time fMRI and its application to neurofeedback. NeuroImage, 62(2), 682-92. doi:10.1016/j.neuroimage.2011.10.009

Weiskopf, N., Sitaram, R., Josephs, O., Veit, R., Scharnowski, F., Goebel, R., ... Mathiak, K. (2007). Real-time functional magnetic resonance imaging: methods and applications. Magnetic Resonance Imaging , 25(6), 989-1003. doi:10.1016/j.mri.2007.02.007

Weiskopf, N., Veit, R., Erb, M., Mathiak, K., Grodd, W., Goebel, R., \& Birbaumer, N. (2003). Physiological self-regulation of regional brain activity using real-time functional magnetic resonance imaging (fMRI): methodology and exemplary data. NeuroImage, 19(3), 577-586. doi:10.1016/S1053-8119(03)00145-9

Wicker, B., Keysers, C., Plailly, J., Royet, J. P., Gallese, V., \& Rizzolatti, G. (2003). Both of us disgusted in My insula: The common neural basis of seeing and feeling disgust. Neuron, 40(3), 655-664. doi:10.1016/S0896-6273(03)00679-2

Wyrwicka, W., \& Sterman, M. B. (1968). Instrumental conditioning of sensorimotor cortex EEG spindles in the waking cat. Physiology \& Behavior, 3(5), 703-707. doi:10.1016/00319384(68)90139-X

Yang, T. T., Menon, V., Eliez, S., Blasey, C., White, C. D., Reid, A. J., ... Reiss, A. L. (2002). Amygdalar activation associated with positive and negative facial expressions. Neuroreport, 13(14), 1737-1741. doi:10.1097/00001756-200210070-00009

Yoo, S.-S., Lee, J.-H., O’Leary, H., Panych, L. P., \& Jolesz, F. A. (2008). Neurofeedback fMRImediated learning and consolidation of regional brain activation during motor imagery. International Journal of Imaging Systems and Technology, 18(1), 69-78. doi:10.1002/ima.20139 
Young, K. D., Zotev, V., Phillips, R., Misaki, M., Yuan, H., Drevets, W. C., \& Bodurka, J. (2014). Real-time FMRI neurofeedback training of amygdala activity in patients with major depressive disorder. PloS One, 9(2), e88785. doi:10.1371/journal.pone.0088785

Zhang, G., Yao, L., Shen, J., Yang, Y., \& Zhao, X. (2014). Reorganization of Functional Brain Networks Mediates the Improvement of Cognitive Performance Following Real-Time Neurofeedback Training of Working Memory. Human Brian Mapping, (December). doi: $10.1002 / \mathrm{hbm} .22731$

Zhang, G., Yao, L., Zhang, H., Long, Z., \& Zhao, X. (2013). Improved working memory performance through self-regulation of dorsal lateral prefrontal cortex activation using realtime fMRI. PloS One, 8(8), e73735. doi:10.1371/journal.pone.0073735

Zhang, G., Zhang, H., Li, X., Zhao, X., Yao, L., \& Long, Z. (2013). Functional alteration of the DMN by learned regulation of the PCC using real-time fMRI. IEEE Transactions on Neural Systems and Rehabilitation Engineering, 21(4), 595-606.

doi:10.1109/TNSRE.2012.2221480

Zilverstand, A., Sorger, B., Sarkheil, P., \& Goebel, R. (2015). fMRI neurofeedback facilitates anxiety regulation in females with spider phobia. Frontiers in Behavioral Neuroscience, 9(June), 1-12. doi:10.3389/fnbeh.2015.00148

Zotev, V., Krueger, F., Phillips, R., Alvarez, R. P., Simmons, W. K., Bellgowan, P., ... Bodurka, J. (2011). Self-regulation of amygdala activation using real-time FMRI neurofeedback. PloS One, 6(9), e24522. doi:10.1371/journal.pone.0024522

Zotev, V., Phillips, R., Young, K. D., Drevets, W. C., \& Bodurka, J. (2013). Prefrontal Control of the Amygdala during Real-Time fMRI Neurofeedback Training of Emotion Regulation. PloS One, 8(11), e79184. doi:10.1371/journal.pone.0079184

Zotev, V., Phillips, R., Yuan, H., Misaki, M., \& Bodurka, J. (2013). Self-regulation of human brain activity using simultaneous real-time fMRI and EEG neurofeedback. NeuroImage, 85, 985-995. doi:10.1016/j.neuroimage.2013.04.126

Zuberer, A., Brandeis, D., \& Drechsler, R. (2015). Are treatment effects of neurofeedback training in children with ADHD related to the successful regulation of brain activity? A review on the learning of regulation of brain activity and a contribution to the discussion on specificity. Frontiers in Human Neuroscience, 9(March), 1-15. doi:10.3389/fnhum.2015.00135 